\title{
Les interférences de l'arabe dans le français parlé par les femmes maghrébines résidant en région parisienne
}

\author{
María Ballarín Rosell ${ }^{1}$
}

Recibido: 10 de septiembre de 2019 /Aceptado: 27 noviembre de 2020

Résumé. Les flux migratoires entrainent un contact étroit entre la langue première de l'immigré et celle du pays d'accueil. Ce contact donne lieu à des interférences entre les deux langues, qui se manifestent souvent par l'apparition de traces de la langue première du migrant dans son discours en langue d'accueil. Le présent article propose une analyse des interférences de l'arabe repérées dans le discours de 8 femmes d'origine maghrébine qui habitent à Paris ou dans ses alentours, de même qu'une brève analyse du rapport entre ces interférences et le sentiment d'insécurité linguistique.

Mots clés: interférence, transfert linguistique, contact de langues, immigration, insécurité linguistique

\section{[es] Las interferencias del árabe en el francés hablado por las mujeres magrebíes residentes en la región parisina}

Resumen. Los flujos migratorios traen consigo un estrecho contacto entre la lengua de la persona migrante y la del país de acogida. Este contacto da lugar a interferencias entre ambas lenguas, manifestadas frecuentemente bajo la forma de huellas de la lengua materna del migrante en su discurso en la lengua de acogida. El presente artículo propone un análisis de las interferencias del árabe detectadas en el discurso de 8 mujeres de origen magrebí que residen en París o en sus alrededores, así como un breve análisis de la relación entre estas interferencias y el sentimiento de inseguridad lingüística.

Palabras clave: interferencia, transferencia lingüística, lenguas en contacto, inmigración, inseguridad lingüística

\section{[en] Arabic transfer in French spoken by Maghreb women living in the Paris region}

\begin{abstract}
Migration flows come with a tight contact between the immigrant's first language and the language of the host country. This contact leads to interferences between both languages, usually revealed as fingerprints of the immigrant's mother tongue in their speech in the host-country language. This paper presents a study of the interferences from Arabic detected on the speech of 8 Maghreb women who live in Paris and its surroundings, as well as a brief analysis of the relation between those interferences and the feeling of linguistic insecurity.
\end{abstract}

Keywords: interference, linguistic transfer, language contact, immigration, linguistic insecurity.

Sommaire: 1 . Introduction ; 2. Le phénomène de l'interférence ; 3. Analyse du corpus ; 4. L'interférence comme indice et déclencheur d'insécurité linguistique ; 5 . Conclusion; 6. Conventions de transcription

$1 \quad$ E-mail: maria.ballarin@hotmail.com 
Cómo citar: Ballarín Rosell, M. (2021) : Les interférences de l'arabe dans le français parlé par les femmes maghrébines résidant en région parisienne, en Anaquel de Estudios Árabes, 32, 35-52.

\section{Introduction}

Le français et l'arabe entretiennent, depuis les mouvements colonisateurs de la France en Afrique du Nord au cours du XIX et du XX ${ }^{\mathrm{e}}$ siècle, une relation étroite et souvent sensiblement paradoxale. Au Maghreb, même si l'indépendance des colonies a marqué une volonté de rupture avec la langue française, celle-ci a gardé une place importante dans certains domaines -notamment dans l'éducation et l'administration- étant toujours la langue de prestige socio-économique ${ }^{2}$. Le Maghreb comptait ainsi en 2016 32,5 millions de francophones ${ }^{3}$. En France, la présence maghrébine est remarquable, représentant près d'un tiers du total de la population immigrée ${ }^{4}$.

Les situations de contact entre ces deux langues sont donc fréquentes aussi bien en territoire maghrébin que français et donnent lieu à des phénomènes dérivés de l'interaction de langues tels que le code-switching ou l'interférence linguistique. Nous nous sommes intéressées dans notre étude par ce dernier, le code-switching ayant déjà été abondamment étudié. On peut citer, à titre d'exemple, la contribution de Karima Ziamari, qui réalise, dans un ouvrage intitulé Le code switching au Maroc, une analyse morphosyntaxique du code-switching arabe marocain-français à partir d'un corpus de conversations bilingues d'étudiants marocains ${ }^{5}$. D'autres chercheurs tels que Caubet ${ }^{6}$ ou Barontini $^{7}$ ont étudié ce même phénomène sur le territoire français, prouvant que le français et les différentes variétés de l'arabe maghrébin demeurent en quelque sorte indissociables chez la population maghrébine résidant en France.

Le concept d'interférence a été traité depuis le milieu du siècle dernier par de nombreux chercheurs de renom tels que Weinreich, Mackey ou Grosjean, puis plus récemment par d'autres spécialistes en bilinguisme comme Lüdi et $\mathrm{Py}^{8}$ ou Jarvis et Pavlenko. Plus spécifiquement, les interférences de l'arabe avec le français sont abordées par plusieurs auteurs (Calaque, Nissabouri, Hamida et Baidoun, entre autres), dont la plupart se concentrent exclusivement sur l'aspect phonologique.

2 SAYAHI, Lotfi, «French in the Maghreb », dans Diglossia and Language Contact. Language Variation and Change in North Africa (2014) 38-46, 42.

3 ORGANISATION INTERNATIONALE DE LA FRANCOPHONIE, (2016) Qui parle français dans le monde? [En ligne], disponible sur http://observatoire.francophonie.org/qui-parle-francais-dans-le-monde/ (Dernier accès le 10 septembre 2020 ).

4 INSEE, (2019) Immigrés, étrangers [En ligne], disponible sur https://www.insee.fr/fr/statistiques/3633212\#consulter (Dernier accès le 10 septembre 2020).

5 Cf. ZIAMARI, Karima, Le code switching au Maroc : L'arabe marocain au contact du français, L'Harmattan, Paris 2008.

6 Cf. CAUBET, Dominique, « Métissages linguistiques ici (en France) et là-bas (au Maghreb) », dans VEI Enjeux, 130 (2002), 117-132.

7 Cf. BARONTINI, Alexandrine, «Alternance codique arabe algérien / français, en France : négociations à partir d'une consigne donnée par la recherche », dans Estudios de dialectología norteafricana y andalusí 10 (2006), 69-80.

8 Cf. LÜDI, Georges \& PY, Bernard, Être bilingue, 4e édition, Peter Lang, Berne 2013 [1 $1^{\text {ère }}$ édition : 1986]. Le reste des auteurs mentionnés dans ce paragraphe seront cités plus tard et le lecteur pourra trouver la référence en bas de page. 
Tenant compte de tous les domaines de la langue et non seulement du phonologique, nous présenterons dans cet article une analyse linguistique des interférences de l'arabe observées dans le discours oral de huit femmes d'origine maghrébine nées au Maghreb- résidant actuellement en région parisienne.

Ensuite, un regard plus sociolinguistique sera porté sur ce phénomène, tout en analysant son rapport avec le sentiment d'insécurité linguistique, qui apparait " lorsque les locuteurs considèrent leur façon de parler comme peu valorisante et ont en tête un autre modèle, plus prestigieux, mais qu'ils ne pratiquent pas $"{ }^{9}$. Cette notion, exposée pour la première fois par $\mathrm{Labov}^{10}$, est étudiée ultérieurement par d'autres auteurs de grand renom comme Calvet ${ }^{11}$ ou Bourdieu ${ }^{12}$. Labov et Calvet analysent le phénomène de l'hypercorrection ${ }^{13}$ comme l'une des conséquences du sentiment d'insécurité. D'autres linguistes contemporains ont mené des recherches sur l'insécurité linguistique (Michel Francard, Aude Bretegnier, Gudrun Ledegen et Didier de Robillard, entre autres). L'apparition de ce sentiment chez les femmes d'origine maghrébine en France a été analysée par Luc Biichlé en 2011 ${ }^{14}$.

\section{Le recueil des données}

Notre corpus se constitue de huit entretiens individuels d'environ 35 minutes enregistrés au magnétophone (au total environ 275 minutes d'enregistrement), réalisés entre février et mars 2020 dans le cadre d'une étude sur les pratiques et les représentations langagières des femmes d'origine maghrébine résidant en région parisienne.

Les critères que nous avons suivis pour la recherche de nos informatrices sont les suivants :

Sexe : féminin

Origine : nées au Maghreb et immigrées en France à l'âge adulte

Langue première : arabe

Âge : adulte

Lieu de résidence : Paris et ses alentours

Temps passé en France : plus de 5 ans

Niveau de français : suffisant pour répondre à nos questions

Suivant ces constants, nous avons essayé de constituer un corpus le plus varié possible quant à l'âge et au pays d'origine, ainsi qu'au niveau d'études, tout en cherchant à obtenir un nombre plus ou moins équilibré d'informatrices avec des études universitaires et sans études universitaires.

9 Cf. CALVET, Louis-Jean, La sociolinguistique, Presses Universitaires de France, Paris 1993, 50.

10 Cf. LABOV, William, Sociolinguistic Patterns, University of Pennsylvania Press, Pennsylvania 1972 ; LABOV, William, Sociolinguistique, Les Éditions de Minuit, Paris 1976.

11 Cf. CALVET, La sociolinguistique.

12 Même si Bourdieu n'utilise pas le terme d'insécurité linguistique, il aborde les conséquences du sentiment d'infériorité (de la classe dominée face à la classe dominante) dans la langue. $C f$. BOURDIEU, Pierre, $C e$ que parler veut dire. L'économie des échanges linguistiques, Fayard, Paris 1982.

13 «Restitution exagérée des formes prestigieuses » engendrée par un « mouvement tendanciel vers la norme » (CALVET, La sociolinguistique, 55).

14 BIICHLÉ, Luc, « Insécurité linguistique et réseaux sociaux denses ou isolants : le cas de femmes maghrébines dans la tourmente », Lidil, nº44 (2011), 13-26. 
Le nombre réduit d'enquêtées faisant partie de notre étude rend possible une petite introduction des profils socioprofessionnels de celles-ci :

\begin{tabular}{|c|c|l|l|c|l|}
\cline { 2 - 6 } \multicolumn{1}{c|}{} & Âge & Pays d'origine & Niveau d'études & $\begin{array}{c}\text { Date } \\
\text { d'arrivée }\end{array}$ & \multicolumn{1}{c|}{ Profession } \\
\hline $\mathbf{N}^{\mathbf{0}}$ & 39 & Algérie & Universitaire & 2005 & Doctorante \\
\hline $\mathbf{N}^{\mathbf{0}}$ & 37 & Libye & Universitaire & 2008 & Enseignante de FLI \\
\hline $\mathbf{N}^{\mathbf{0}}$ & 30 & Algérie & Universitaire & 2011 & Cheffe de projet \\
\hline $\mathbf{N}^{\mathbf{0}}$ & 57 & Tunisie & Non universitaire & 1987 & Femme de ménage \\
\hline $\mathbf{N}^{\mathbf{0} 5}$ & 37 & Maroc & Sans études & 2002 & Femme de ménage \\
\hline $\mathbf{N}^{\mathbf{0}} \mathbf{6}$ & $\sim 30$ & Libye & Universitaire & 2013 & Esthéticienne \\
\hline $\mathbf{N}^{\mathbf{0}} \mathbf{7}$ & 50 & Maroc & Non universitaire & 1990 & Agente administrative \\
\hline $\mathbf{N}^{\mathbf{0} 8}$ & 50 & Algérie & Universitaire & 1994 & Professeure de secondaire \\
\hline
\end{tabular}

Quant à leur rapport avec la langue française, la plupart des informatrices l'ont apprise en tant que langue étrangère, que ce soit pendant leur scolarisation dans leur pays d'origine $\left(n^{\circ} 1,2,4,6\right.$ et 7) ou déjà en France par immersion $\left(n^{\circ} 5\right)$. Pour la $n^{\circ} 8$, le français est sa langue première avec l'arabe algérien, car sa mère était Pied-Noire française et a toujours communiqué avec elle en français. Le cas de l'enquêtée $n^{\circ} 3$ est particulier, car le français n'est pas sa langue maternelle, mais une partie de sa famille a toujours habité en France et elle y allait souvent en vacances et communiquait avec sa famille en français depuis très petite, c'est pourquoi son français est impeccable.

Le but des entretiens étant d'analyser aussi bien la forme que le contenu du discours des enquêtées, nous avons opté pour l'entretien semi-directif : partant d'une liste de questions préétablie, nous avons essayé de créer un dialogue naturel avec les informatrices où elles pouvaient parler en toute liberté. Nos questions portaient principalement sur leur expérience migratoire, leur sentiment identitaire, leurs habitudes langagières et leur propre perception de leur français (compétences, accent, sûreté...).

Les entretiens se sont pour la plupart déroulés dans des endroits publics (sur le lieu de travail de l'enquêtée ou dans un café), à l'exception des entretiens avec les informatrices $n^{\circ} 8$ et 9 , qui ont eu lieu dans son domicile et par téléphone respectivement. Toutes les informatrices étaient conscientes avant l'entretien que celui-ci serait utilisé pour étudier leur usage du français et le rapport de ce dernier avec l'expérience migratoire. Cependant, elles n'ont pas été prévenues sur le fait que leur discours allait être analysé d'un point de vue linguistique, afin d'éviter, dans la mesure du possible, qu'elles modifient volontairement leur parler naturel. 


\section{Le phénomène de l'interférence}

Tout au long du processus d'acquisition de n'importe quelle langue seconde (L2), l'apprenti produira des déviations par rapport à la norme. Très souvent, celles-ci seront issues de l'influence d'un autre code, généralement celui de sa langue première (L1). Ces déviations reçoivent le nom d'interférences et celles-ci perdureront dans le discours du bilingue même après avoir acquis une très bonne maîtrise de la L2. Ces interférences peuvent toutefois être bidirectionnelles : non seulement la L1 peut interférer dans la production de la L2, mais aussi inversement. C'est ainsi que Mackey définit l'interférence comme un « écart de la norme locale dû à l'insertion dans le message d'éléments provenant d'un autre code $»^{15}$. Grosjean maintient cet aspect bidirectionnel mais y ajoute le caractère involontaire, afin de distinguer l'interférence d'autres phénomènes conscients et contrôlés tels que l'emprunt ou le code-switching ${ }^{16}$.

Les interférences ont pendant longtemps été perçues comme indicateurs de manque d'intelligence et d'étroitesse d'esprit, parmi d'autres associations aussi négatives ${ }^{17}$. Ce n'est qu'au milieu du $\mathrm{XX}^{\mathrm{e}}$ siècle que ces idées vont être contestées par plusieurs auteurs, dont Weinreich ${ }^{18}$. Dès lors, l'interférence va être considérée comme une caractéristique inévitable de l'apprentissage et de l'usage d'une langue ${ }^{19}$.

Nombreuses sont les catégorisations qui ont été proposées dans l'étude des interférences langagières. Nous nous intéresserons dans ce travail à la classification par domaines linguistiques recueillie par Jarvis et Pavlenko. Neuf types d'interférences seraient ainsi distinguées : phonologiques, orthographiques, lexicales, sémantiques, morphologiques, syntaxiques, discursives, pragmatiques et sociolinguistiques. Or, seule quatre catégories seront décrites en détail et étudiées dans ce travail dans le but d'alléger notre analyse et de l'adapter aux caractéristiques de notre corpus. Nous analyserons ainsi les interférences phonologiques, lexico-sémantiques, morphologiques et syntaxiques de l'arabe dans le français de nos informatrices. Nous allons introduire ici brièvement ces catégories en présentant les notions de base concernant le phénomène de l'interférence en général et les interférences de l'arabe ${ }^{20}$ en français en particulier. Notre but n'est pas celui de procurer une liste exhaustive des interférences possibles, mais d'offrir un cadre de référence qui serve d'introduction à l'analyse des interférences repérées dans notre corpus.

\section{Les interférences phonologiques}

Dans le discours des migrants arabophones en France, les interférences phonologiques sont celles qui seront repérées tout de suite, y compris par les non-spécialistes. « En écoutant certains locuteurs arabophones, l'auditeur francophone naïf re-

15 MACKEY, William Francis, Bilinguisme et contact des langues, Éditions Klincksieck, Paris 1976, 414.

16 Cf. GROSJEAN, François, Life with two languages. An introduction to bilingualism, Harvard University Press, Harvard 1982.

17 Cf. JARVIS, Scott \& PAVLENKO, Aneta, Crosslinguistic Influence in Language and Cognition, Routledge, New York 2007, 2.

18 Cf. WEINREICH, Uriel, Languages in contact : Findings and Problems 1953 (version consultée : KOHLHASE, Jörg [trad.], Sprachen in Kontakt, Beck, Munich 1977).

19 Cf. JARVIS \& PAVLENKO, Crosslinguistic Influence, 3.

20 Les Maghrébins peuvent avoir pour L1 l'arabe, le berbère ou les deux. Or, presque la totalité de nos enquêtées sont exclusivement arabophones, donc nous n'allons pas tenir compte des éventuelles interférences du berbère. 
connaît ce qu'il appelle un accent arabe qui constitue une variété d'accent étranger $»^{21}$. Calaque reconnaît que l'empreinte de la L1 du locuteur est difficilement annulable, même chez les locuteurs confirmés.

D'après Weinreich, à chaque fois qu'il y a une différence entre deux systèmes phonologiques, il y aura aussi une interférence ${ }^{22}$ et, pour les arabophones, l'apprentissage de la langue française exige «l'acquisition d'un système phonétique et phonologique radicalement différent de celui de leur langue naturelle $»^{23}$. En effet, de nombreux phonèmes du français ne sont pas présents dans la phonologie arabe, notamment en ce qui concerne les voyelles.

Dans le système phonétique arabe, les voyelles se distinguent par deux traits : leur durée (elles peuvent être longues, mi-longues ou brèves) et leur timbre ; il existe essentiellement trois timbres $(/ \mathrm{a} /, / \mathrm{u} /$ et $/ \mathrm{i} /)$ complétés par des voyelles intermédiaires ${ }^{24}$ qui, en fonction du dialecte, agissent en tant que phonèmes ou en tant qu'allophones des autres phonèmes. Ainsi, au Maroc, le système phonétique le plus répandu ne comporte que 5 phonèmes vocaliques : les trois voyelles longues correspondant aux trois timbres essentielles (/a:/, /u:/ et /i:/) et deux brèves $(/ / / \text { et } / \mathrm{u} /)^{25}$. L'algérien compte les mêmes voyelles longues et rajoute deux phonèmes vocaliques brefs $(/ \mathrm{a} / \mathrm{et} / \mathrm{i} /)^{26}$ et le dialecte libyen comporte les mêmes voyelles brèves que le marocain mais rajoute deux phonèmes vocaliques longs (/e:/ et /o: $/)^{27}$. Le locuteur arabophone doit donc passer d'un système relativement simple au complexe système vocalique français, composé de 15 phonèmes dont la durée ne suppose pas un trait distinctif ${ }^{28}$. Cela donne lieu à de nombreuses erreurs dont la plupart peuvent être considérées comme des interférences, car le phonème produit appartiendra au système arabe.

Concernant les consonnes, de nombreux phonèmes de l'arabe sont étrangers à la langue française, notamment aux points d'articulation vélaire, uvulaire, pharyngal et laryngal, ainsi que des variantes emphatiques de plusieurs phonèmes partagés par les deux langues. Au contraire, la plupart des phonèmes français existent aussi en arabe maghrébin, à l'exception de l'uvulaire fricative $/ \mathrm{R} /$ (dont il existe en arabe un phonème vélaire très proche $: / \mathrm{y} /$ ) et des nasales $/ \mathrm{n} /$ et $/ \mathrm{n} /$. Les erreurs des arabophones avec les consonnes du français seront donc moins fréquentes, mais la similitude entre les deux codes rend aussi possible l'interférence. Un phénomène récurrent consiste à donner aux consonnes françaises « une prononciation plus 'lourde', plus voilée qui rappelle à beaucoup d'égards l'imprégnation emphatique [de certaines consonnes arabes] ${ }^{29}$.

21 CALAQUE, Élizabeth, « Les erreurs persistantes dans la production de locuteurs arabophones parlant couramment le français "), dans L'information grammaticale n54 (1992) 48-51, 48 (DOI : https://doi.org/10.3406/ igram.1992.3200).

22 Cf. WEINREICH, Languages in Contact, 40.

23 MAUME, Jean-Louis, «L'apprentissage du français chez les Arabophones maghrébins (diglossie et plurilinguisme en Tunisie) », dans Langue française $n^{\circ} 19$, Phonétique et phonologie (1973) 90-107, 90 (DOI : https:// doi.org/10.3406/lfr.1973.5642)

24 Cf. MARÇAIS, Philippe, Esquisse grammaticale de l'arabe maghrébin, Librairie d'Amérique et d'Orient, Paris 1977, 5-6.

25 Cf. AGUADÉ, Jordi, « Árabe marroquí (Casablanca)», dans Manual de dialectología neoárabe (2008), 281-310.

26 Cf. GRAND'HENRI, Jacques, Le parler arabe de Cherchell (Algérie), Institut Orientaliste de Louvain, Louvain-la-Neuve 1972, 17-24

27 Cf. PEREIRA, Christophe, «Le parler arabe de Tripoli (Lybie) », dans Estudios Árabes e Islámicos, Estudios de dialectología árabe 4 (2010), 23-82.

28 CALAQUE, « Les erreurs persistantes », 48-49.

29 NISSABOURI, Abdelfattah, Le français tel qu'on le prononce à Casablanca. Reflets des tendances actuelles de l'arabe marocain sur la prononciation du français, Linguistique, Université de Rennes 2 1994, 2 [En ligne], disponible sur https://tel.archives-ouvertes.fr/tel-00657638 (Dernier accès le 10 septembre 2020). 
Finalement, l'influence de la L1 peut être aperçue à d'autres niveaux phonologiques tels que l'intonation, l'accentuation ou le rythme ${ }^{30}$, ainsi que dans la structure syllabique.

\section{Les interférences lexico-sémantiques}

Dans le domaine lexical, les interférences les plus communes sont les faux amis, les traductions mot à mot et les créations dans une langue sur le modèle de l'autre ${ }^{31}$. Lorsque les langues sont typologiquement éloignées, il est fréquent d'utiliser des termes existant dans la langue cible, mais avec une signification qui reflète l'influence sémantique de l'autre langue ${ }^{32}$, comme dans les calques sémantiques.

Le choix des mots est souvent fortement influencé par la L1, ce qui peut se manifester aussi bien par le type de mots choisi que par les mots en soi, de même que par leur fréquence ${ }^{33}$.

Taghrid Baidoun recueille les données présentées par plusieurs chercheurs sur cet aspect et conclut que les domaines où le discours des arabophones présente le plus d'influences lexico-sémantiques de l'arabe sont la synonymie (notamment lorsqu'il existe un seul mot en arabe pour plusieurs équivalents en français) et l'usage erroné de certains adjectifs qualificatifs et d'emprunts d'autres langues (qui ne s'utilisent pas en français ou qui n'ont pas le même sens) $)^{34}$.

\section{Les interférences morphologiques}

Les identifications ou les associations des structures morphologiques des deux langues mènent souvent à des interférences concernant la compréhension ou la production chez les utilisateurs de la L $2^{35}$. L'un des aspects les plus problématiques à cet égard est l'expression du temps et de l'aspect verbal ${ }^{36}$.

Les interférences les plus fréquentes des arabophones dans le domaine morphologique s'observent dans le choix du genre des noms et dans l'utilisation de la forme pronominale du verbe à la place de sa forme non pronominale ${ }^{37}$.

\section{Les interférences syntaxiques}

Même si les chercheurs ont pendant longtemps affirmé que la syntaxe était immunisée contre le phénomène des interférences, les études les plus récentes ont démontré le contraire ${ }^{38}$. En effet, ce type d'interférences est fréquent chez les francophones d'origine maghrébine.

\footnotetext{
Cf. JARVIS \& PAVLENKO, Crosslinguistic influence, 67.

CALVET, Louis-Jean, La sociolinguistique, Presses Universitaires de France, Paris 1993, 25.

JARVIS \& PAVLENKO, Crosslinguistic influence, 75-77.

JARVIS \& PAVLENKO, Crosslinguistic influence, 91-92.

BAIDOUN, Taghrid, L'influence de la langue arabe sur le français des arabophones migrants, Linguistique, Université de Rennes II 2015 [En ligne], disponible sur https://dumas.ccsd.cnrs.fr/dumas-01427030 (Dernier accès le 10 septembre 2020).

35 JARVIS \& PAVLENKO, Crosslinguistic influence, 93.

36 JARVIS \& PAVLENKO, Crosslinguistic influence, 94.

37 BAIDOUN, L'influence de la langue arabe, 35.

38 JARVIS \& PAVLENKO, Crosslinguistic influence, 96.
} 
L'interférence la plus évidente s'observe au niveau de l'ordre des mots ou des constituants de la phrase, mais il existe aussi d'autres domaines où les interférences sont récurrentes en raison des différences entre les normes grammaticales des deux codes. L'accord, l'utilisation des pronoms (notamment les relatifs) et le choix des prépositions se trouvent parmi ces domaines ${ }^{39}$.

\section{Analyse du corpus}

Nous nous proposons de présenter ici toutes les interférences de l'arabe que nous avons pu repérer dans le discours de nos enquêtées, suivant la classification par domaines linguistiques qui vient d'être introduite. Nous essayerons de fournir les explications nécessaires sur la phonétique et la morphologie arabes pour comprendre l'interférence en nous appuyant sur les études déjà publiées dans ce domaine et sur une grammaire de l'arabe maghrébin. Nous avons consulté également plusieurs experts en dialectologie arabe pour obtenir des informations plus spécifiques sur les dialectes de nos enquêtées. L'auteure de chaque mot ou énoncé présentant une interférence sera indiquée entre parenthèses.

Il faut préciser avant tout que, étant donné la situation diglossique du Maghreb, les interférences se produiront toujours entre le français et les variétés vernaculaires ou dialectales de l'arabe propres à chaque pays, sans tenir compte de l'arabe standard, qui n'est pas une langue parlée ${ }^{40}$. Or, l'arabe standard et le dialectal maghrébin partagent certaines caractéristiques et certains éléments du lexique, c'est pourquoi nous emploierons souvent le terme arabe pour désigner l'ensemble des variétés (écrite et parlées). Lorsque la caractéristique présentée sera commune à toutes les variétés dialectales de l'arabe au Maghreb, mais pas à l'arabe standard, nous parlerons d'arabe maghrébin. Finalement, lorsque nous ferons référence à un dialecte concret, ceci sera précisé.

Enfin, nous voudrions remarquer que, d'après Weinreich, les interférences entre les langues sont généralement moins fréquentes lorsque le bilingue communique avec une personne monolingue ${ }^{41}$. Nous pouvons donc supposer que le nombre d'interférences présentes dans leur parler est plus large lorsque nos informatrices communiquent en français avec d'autres arabophones, ce que nous n'avons pas pu vérifier dans cette étude.

\section{Interférences phonologiques}

Comme nous l'avons déjà annoncé précédemment, les systèmes phonologiques arabe et français sont assez éloignés, ce qui donne lieu à de nombreuses interfé-

39 Cf. BEN AMOR BEN HAMIDA, Thouraya, « Erreurs interférentielles arabe-français et enseignement du français », Synergies Tunisie nº1 (2009) 105-117 [En ligne], disponible sur https://gerflint.fr/Base/Tunisie1/tunisie1. html (Dernier accès le 10 septembre 2020).

40 La constatation que ce sont les variétés vernaculaires de l'arabe qui doivent être prises en compte dans l'étude des phénomènes de contact de langues a déjà été faite lors des études interférentielles entre l'arabe andalou et les langues romanes dans la Péninsule Ibérique (cf. VICENTE, Ángeles, El proceso de arabización de Alandalús: un caso medieval de interacción de lenguas, Instituto de Estudios Islámicos y del Oriente Próximo, Zaragoza 2006).

41 Cf. WEINREICH, Languages in Contact. 
rences. Il s'agit en effet du domaine qui a été le plus largement étudié par les chercheurs.

Plusieurs phonèmes vocaliques du français n'existent pas comme tels dans le système arabe, même s'ils peuvent y être présents en tant qu'allophones d'autres phonèmes. Ceci provoque une forte confusion chez l'arabophone, qui tend à échanger les phonèmes du français, croyant que cela n'affecte pas le sens du mot, comme c'est le cas en arabe. Nous allons expliquer en détail ci-dessous les déviations dans la prononciation des voyelles que nous avons repérées dans notre corpus et qui proviennent de ces interférences :

Prononciation des phonèmes /e/, / / / et /y/ comme $/ \mathrm{i} /{ }^{42}\left(\mathrm{n}^{\mathrm{o}} 1,2,4,5,6,7\right)$

Exemples : aidé [ide] $\left(\mathrm{n}^{\circ} 2\right)$, échange [ifãz] $\left(\mathrm{n}^{\circ} 1\right)$, bénévole [binivol] $\left(\mathrm{n}^{\mathrm{o}} 1\right)$, bêtise [bitiz] $\left(\mathrm{n}^{\circ} 1\right)$, caissière [kisjeR] $\left(\mathrm{n}^{\circ} 7\right)$; elle [il] $\left(\mathrm{n}^{\circ} 4,5,6,7\right)$, dès [di] $\left(\mathrm{n}^{\circ} 1\right)$; suffisant [sifizã] $\left(\mathrm{n}^{\circ} 1\right)$, bureau [biRo] $\left(\mathrm{n}^{\circ} 4\right)$

Prononciation des phonèmes $/ \varnothing /$ et $/ \mathrm{u} /$ comme $/ \mathrm{o} /\left(\mathrm{n}^{\mathrm{o}} 2,5,7\right)$

Exemples : veut [vo] $\left(\mathrm{n}^{\circ} 2\right)$, peut/peux $[\mathrm{po}]\left(\mathrm{n}^{\circ} 2,5\right)$, eux $[\mathrm{o}]\left(\mathrm{n}^{\circ} 2,7\right) ;$ couleur $\left[\right.$ kolœR] $\left(\mathrm{n}^{\circ} 2\right)$

Difficultés avec les nasales $\left(\mathrm{n}^{\circ} 2,4,6\right)$ : oralisation ou confusion des deux nasales.

Exemples : enseigner [õsene] (n²), mutation [mytasjo] (n4), A1 [a'a] (nº6)

Le système consonantique arabe influence également la production des consonnes françaises, comme nous pouvons l'apprécier dans les exemples suivants, extraits de notre corpus :

Remplacement de /p/ par /b/ $\left(\mathrm{n}^{\circ} 2,6\right)$ : le phonème /p/ est étranger à l'arabe, mais il peut « figurer dans des prononciations altérées, ou dans des mots d'emprunt ${ }^{43}$. Il est en tout cas beaucoup moins fréquent en arabe que /b/, ce qui explique l'interférence.

Exemples : pour [buR] $\left(\mathrm{n}^{\circ} 6\right)$, groupe $[\mathrm{gRub}]\left(\mathrm{n}^{\circ} 6\right), C A P[\mathrm{kab}]\left(\mathrm{n}^{\circ} 6\right)$, depuis [dəbui] $\left(\mathrm{n}^{\circ} 2\right)$, exemple [Egzãbl] $\left(\mathrm{n}^{\circ} 2\right)$

Remplacement de /b/ par /p/ (nº $1,5,6,8)$ : il s'agit d'une hypercorrection provoquée par l'interférence expliquée ci-dessus. La locutrice abuse du phonème qui est normalement source de difficultés car elle veut éviter de commettre l'erreur.

Exemples : arabe [aRap] $\left(\mathrm{n}^{\circ} 1,8^{44}\right)$, poubelle [pupcl] $\left(\mathrm{n}^{\circ} 6\right)$, bizarre [pizaR] $\left(\mathrm{n}^{\mathrm{o}} 5\right)$

42 Au nord du Maroc et dans d'autres régions nord-africaines, [i] et [ə] constituent en effet des allophones du même phonème et sont donc interchangeables dans certains contextes. Cf. HEATH, Jeffrey, Jewish and Muslim dialects of Moroccan Arabic, RoutledgeCurzon, New York 2002, p. 188.

43 MARÇAIS, L'arabe maghrébin, 4. En effet, il est fréquent d'entendre le phonème /p/ dans les variétés maghrébines en raison de l'influence d'autres langues telles que le français ou le berbère.

44 Il faut noter que, dans le discours de l'enquêtée $n^{\circ} 8$, seulement deux interférences de l'arabe ont pu être remarquées, la seconde étant décrite un peu plus tard (remplacement de /g/ par /q/ dans le mot langue). 
Prononciation roulée de l'uvulaire française $/ R /\left(n^{\circ} 4,6\right)$ : même si les arabophones possèdent dans leur système un phonème très proche $/ \mathrm{\gamma} /$, ils l'assimilent parfois à leur $/ \mathrm{r} /{ }^{45}$.

Exemples : première [prəmjer] $\left(\mathrm{n}^{\circ} 4\right)$, par exemple [par cgzãpl] $\left(\mathrm{n}^{\circ} 4\right)$, comprends [kõprã] (nº 4$)$, peut-être [pøtctr] (nº)

Remplacement des phonèmes liquides $\left(n^{\circ} 5\right)$ : en arabe maghrébin, les liquides /1/ et $/ \mathrm{n} /$ 《 sont particulièrement sujets à des mutations ${ }^{46}$. Ceci pourrait influencer leur prononciation en français.

\section{Exemple : prononcer [pRolõse] (n5)}

D’autres phénomènes phonétiques ont été repérés dans le discours de nos informatrices qui pourraient relever directe ou indirectement d'interférences de l'arabe. Il s'agit de deux types de métaplasmes : 1'aphérèse ${ }^{47}$ et l'épenthèse ${ }^{48}$.

Aphérèse provoquée par une analyse erronée de la partie supprimée :

- imaginez prononcé [mazine] (nº) : possible association de [i] avec le préfixe de la troisième personne en arabe. La locutrice l'enlèverait par intuition parce qu'il ne correspondrait pas à la deuxième personne de la forme exprimée ${ }^{49}$.

- informations prononcé [foRmasjõ] $\left(\mathrm{n}^{0} 5\right)$ : tenant compte de la confusion entre les différentes voyelles nasales, notre enquêtée aurait pu interpréter la première syl-

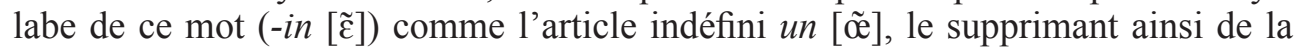
forme plurielle du mot. Cette possibilité gagne du poids si l'on tient compte du fait que l'enquêtée qui a commis cette erreur est illettrée et ne connaît donc pas la forme écrite du mot ${ }^{50}$.

Épenthèse provoquée par une hypercorrection : les voyelles brèves disparaissent en arabe maghrébin lorsqu'elles ne sont pas « indispensables à l'articulation des groupes de consonnes formant syllabe $\rangle^{51}$, c'est pourquoi elles tendent à disparaître en syllabe ouverte. La prononciation des exemples suivants pourrait relever d'une

45 BAIDOUN, L'influence de la langue arabe, 21.

46. MARÇAIS, L'arabe maghrébin, 22.

47 «L'aphérèse est un changement phonétique qui consiste en la chute d'un phonème initial ou en la suppression de la partie initiale (une ou plusieurs syllabes) d'un mot» (DUBOIS, J., GIACOMO, M., GUESPIN, L., MARCELLESI, C., MARCELLESI, J.B. \& MÉVEL, J.P., Dictionnaire de linguistique, Larousse, Paris 2002 [1 ${ }^{\text {ère }}$ édition : 1994], 43).

48 «On appelle épenthèse le phénomène qui consiste à intercaler dans un mot ou un groupe de mots un phonème non étymologique pour des raisons d'euphonie, de commodité articulatoire, par analogie, etc. » (DUBOIS et al., Dictionnaire de linguistique, 483).

49 Il faut préciser que cette formule n'a été utilisée que par une de nos informatrices, c'est pourquoi nous ne pouvons pas certifier qu'il s'agisse d'une erreur fréquente chez la population arabophone. L'explication que nous proposons ne constitue donc qu'une hypothèse.

50 Des exemples similaires d'aphérèses dérivées d'une interférence peuvent être retrouvés dans certains mots en arabe marocain empruntés au français et à l'espagnol, où la première syllabe du mot original disparaît parce qu'elle est assimilée à tort à l'article arabe : bart $m a$ : (du français « appartement »), bu:ga:du (de l'espagnol « abogado »-avocat-). Cf. BALLARÍN, María, Croisement des cultures et des langues. La place du français au sein du marché sociolinguistique marocain, Zaragoza 2018, 23.

51 MARÇAIS, L'arabe maghrébin, 12. 
volonté de récupération d'une voyelle qu'elles pensent avoir éliminée, provoquant ainsi une prononciation hypercorrecte ${ }^{52}$.

Exemples : explique [ckseplik] $\left(\mathrm{n}^{\circ} 6\right)$, expressions [cksepResjõ] $\left(\mathrm{n}^{\circ} 6\right)$, exemple [ckezãpl] $\left(\mathrm{n}^{\circ} 2\right)$

\section{Interférences lexico-sémantiques}

Certaines erreurs de lexique que nos informatrices ont commises au cours des entretiens peuvent être considérées comme des interférences de leur L1. Les calques sémantiques et la synonymie sont à l'origine de la plupart de ces interférences.

Ainsi, les adverbes très et trop et les verbes lâcher et relâcher, par exemple, sont perçus par nos informatrices comme des synonymes, car ils n'ont qu'un seul équivalent en $\operatorname{arabe}^{53}$, ce qui donne lieu à des confusions comme les suivantes :

1. « [...] sinon à la maison on parlait arabe dialectal, arabe algérien, euh... quelques mots de français bien sûr qu'elles... qu'elles sont présents trop dans nos dialectes $\gg\left(\mathrm{n}^{\mathrm{0}} 1\right)^{54}$

2. « ils m'ont dit à la lettre : on doit pas vous... vous lâcher, d'accord ? vous lâcher ou bien vous relâcher? [rires] C'est ça le problème de l- oui, c'est ça la difficulté ici » $\left(\mathrm{n}^{\circ} 2\right)$

Enfin, deux possibles calques sémantiques de l'arabe ont été repérés dans les formulations produites par nos enquêtées :

1. « j'ai fait double nationalité » $\left(\mathrm{n}^{\circ} 4\right) \rightarrow$ possible calque d'une construction en arabe tunisien avec le verbe Smalt (j'ai fait) et le syntagme la double nationalité en français ${ }^{55}$ : Sməlt la double nationalité.

2. «non, pour moi ça a jamais été difficile parce que franchement la première fois j'ai travaillé comme job d'étudiante [...] » (pour dire au début) $\left(\mathrm{n}^{\circ} 7\right) \rightarrow$ probable calque de l'arabe maghrébin, où la forme $f$-luwwal (au début) inclut l'adjectif $u w w a l$ (premier) substantivé et précédé de la préposition $f$ -

\section{Interférences morphologiques}

En accord avec les propos présentés précédemment, la plupart des interférences morphologiques qui ont été repérées dans notre corpus relèvent du choix du temps verbal et du genre des noms, ainsi que des difficultés avec la forme pronominale des verbes.

En ce qui concerne la conjugaison verbale, il faut préciser que le concept de temps verbal n'existe pas en tant que tel en arabe, où le verbe se présente sous deux

52 Cette explication est également hypothétique, mais le nombre plus large d'exemples de ce phénomène nous encourage à croire qu'il s'agit effectivement d'une interférence récurrente.

53 En arabe algérien, il y a deux adverbes qui peuvent signifier indifféremment très ou trop : bazzāf et yassar. Quant à lâcher et relâcher, leur équivalent en algérien est țlag-yițlag C'est la traduction en algérien qui est donnée dans les deux cas parce que les interférences décrites ci-après ont été produites par deux informatrices d'origine algérienne.

54 Elle ne semble pas vouloir dire que la quantité de mots français soit excessive, mais plutôt abondante.

55 La pratique du code-switching ou alternance codique arabe-français est fréquente dans les pays du Maghreb. 
aspects : l'accompli (si l'action dénotée par le verbe est réalisée) et l'inaccompli ( si cette action n'est pas réalisée $)^{56}$. Cette structuration bien différente de l'expression verbale donne lieu à une forte confusion de la part des arabophones avec les nombreux temps verbaux du français, ce que nous avons pu vérifier lors de nos entretiens.

Exemples :

1. « [...] quelquefois dans mon rêve je parle française, mais française comme dans les journals hein [rires] comme j'aime être, oui » $\left(\mathrm{n}^{\circ} 6\right)$

2. «j'ai déjà deux enfants quand je commence à travailler » $\left(n^{\circ} 4\right)$

3. «j'habite pas ici avant» $\left(\mathrm{n}^{\mathrm{o}} 4\right)$

4. « avant je lis beaucoup, j'ai lu beaucoup » $\left(n^{\circ} 6\right)$

Dans le premier exemple, nous constatons une difficulté à employer le conditionnel, celui-ci étant remplacé par le présent «j'aime » (traduisant l'aspect inaccompli de l'arabe). Dans les exemples 2, 3 et 4, ce sont les temps du passé qui posent un problème pour nos enquêtées, qui ont en général une tendance à parler au présent des actions ou des faits appartenant au passé. Même si, en arabe, ces faits seraient exprimés avec la forme accomplie, qu'elles pourraient traduire en français par un temps du passé, la confusion dont leurs propos témoignent à l'égard de ce nouveau système les induit visiblement à éviter tous les temps passés et à simplifier le système en privilégiant le présent.

En outre, l'absence du mode subjonctif en arabe dialectal entraîne son remplacement par l'indicatif dans certains cas où le subjonctif est obligatoire en français.

Exemples :

1. «j'ai oublié qu'il fallait que je traduis en arabe » $\left(\mathrm{n}^{\mathrm{0}} 5\right)$

2. « il faut que je fais $[\ldots] »\left(\mathrm{n}^{\mathrm{o}} 7\right)$

Les propositions conditionnelles irréelles posent aussi un problème pour nos informatrices, puisqu'en arabe, aussi bien le verbe de la proposition principale que celui de la conditionnelle sont exprimés avec l'aspect accompli. Ceci explique l'interférence suivante, qui a été rapidement corrigée par l'enquêtée : «Si j'avais le choix à l'époque, je choisissais choi-si-rait les études » $\left(\mathrm{n}^{\mathrm{0}} 7\right)$.

Enfin, nous avons repéré des interférences dans l'utilisation de la forme infinitive du verbe. L'arabe possède une forme (le masdar) qui se rapproche de l'infinitif français, mais, même si celle-ci procède d'une forme verbale, elle appartient au système nominal et a le statut syntactique d'un substantif ${ }^{57}$. Par ailleurs, le masdar est utilisé en arabe maghrébin avec une moindre fréquence que l'infinitif en français.

Exemples :

1. « je vais me souviendrai $[\ldots] »\left(\mathrm{n}^{\circ} 1\right)$

2. «[...] pour appris la langue française » $\left(\mathrm{n}^{\circ} 6\right)$

3. « j'ai fait un examen pour connaissance mon niveau en fait » $\left(\mathrm{n}^{\circ} 6\right)$

56 Cf. MARÇAIS, L'arabe maghrébin, 35.

57 Cf. MARÇAIS, L'arabe maghrébin, 83. 
Nous observons donc dans les trois exemples qu'il y a une difficulté à employer l'infinitif. Dans le dernier, d'ailleurs, l'interférence du masdar arabe est assez remarquable, car l'enquêtée a remplacé l'infinitif par la forme nominale dérivée du verbe, qui est la deuxième fonction du masdar en arabe.

La forme pronominale du verbe n'existe pas dans le système verbal arabe, qui emploie d'autres recours pour exprimer les actions réflexives et réciproques, c'est pourquoi cette forme est parfois une source de difficultés pour les arabophones, comme nous l'avons pu constater dans nos entretiens.

Exemples :

1. « je suis pas vraiment à l'aise comme je suis à l'aise en arabe, j'exprime mais avec fluidité $»\left(\mathrm{n}^{\mathrm{o}} 1\right)$

2. «à moi-même je parle en français » $\left(n^{\circ} 5\right)$

3. « [...] j'ai la voix qui... qui... qui, qui tremble, qui se tremble » $\left(\mathrm{n}^{\circ} 2\right)$

Étant donné le caractère arbitraire du genre des noms, il n'est pas surprenant que celui-ci suppose une difficulté pour les apprenants de n'importe quelle langue possédant un genre grammatical. Le cas des arabophones avec le français n'en constitue pas une exception, comme l'a déjà remarqué Hamida ${ }^{58}$. Dans notre corpus, nous avons trouvé de nombreuses interférences de genre, souvent identifiées chez les adjectifs qui accompagnent ces noms ou chez les pronoms de reprise.

Exemples $^{59}$ :

1. « des vocabulaires françaises »; « toutes les vocabulaires » $\left(\mathrm{n}^{\circ} 6\right) \rightarrow$ vocabulaire en arabe est mufrada:t (f. pl.) $)^{60}$

2. « des liens sociales » $\left(\mathrm{n}^{0} 1\right) \rightarrow$ lien en arabe est Sla:qa $(\mathrm{f} .)^{61}$

3. « je parle française » $\left(\mathrm{n}^{\circ} 6\right)$; « c'est pas que je commence à perdre l'arabe, non, je la perds pas » $\left(\mathrm{n}^{\circ} 7\right) \rightarrow$ les noms des langues en arabe sont toujours féminins

4. « un diplôme française » $\left(\mathrm{n}^{\circ} 6\right) \rightarrow$ diplôme en arabe est faha:da (f.)

5. « l'accent est très bien, très belle »; « un accent française »; « vous avez un joli accent, gardez-la » $\left(\mathrm{n}^{\circ} 6\right) \rightarrow$ accent en arabe est lahza (f.)

6. « des mots de français qu'elles sont présents trop [...]» $\left(\mathrm{n}^{\circ} 1\right) \rightarrow$ mots en arabe est kəlma:t (f.)

7. « mon niveau est XXX, elle est pas bien » $\left(\mathrm{n}^{\circ} 6\right) \rightarrow$ niveau en arabe est mustawwa (m.), mais comme il se termine en -a, il est souvent confondu par les propres arabophones avec un nom féminin. Il s'agit donc d'une interférence provoquée par une confusion très fréquente en arabe maghrébin.

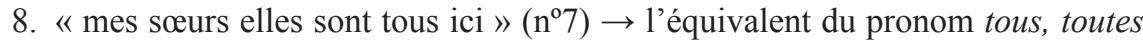
en arabe maghrébin ne distingue pas en genre, il est toujours kullhum (m. et f.)

Le choix erroné de l'article masculin ou féminin pour accompagner un mot qui a en réalité le même genre grammatical en arabe est provoqué par une interférence

58 Cf. HAMIDA, « Erreurs interférentielles arabe-français ».

59 Tous les mots en arabe que nous allons exposer ici ont la même forme dans tous les dialectes, car ce sont des emprunts de l'arabe standard.

${ }_{60}$ Nous pouvons apprécier également une interférence de nombre : comme le mot en arabe est toujours utilisé au pluriel, l'enquêtée utilise en français la forme plurielle.

${ }_{61}$ L'erreur pourrait également provenir d'une difficulté grammaticale inhérente au français, car il s'agit d'un pluriel irrégulier. 
dans la formation de l'article. En arabe, une seule forme englobe toutes les variations de l'article défini français : əl- (qui précède immédiatement le nom qu'il détermine $)^{62}$. L'article indéfini existe en arabe maghrébin comme une « pure innovation dialectale » consistant dans l'utilisation du numéral un (wāh̆ðd) suivi du nom déterminé ${ }^{63}$. L'absence de variation en genre des articles défini et indéfini en arabe provoque visiblement une grande confusion au moment de choisir le genre de l'article en français.

Exemples : « un envie » $\left(\mathrm{n}^{\circ} 1\right)$ « le licence » $\left(\mathrm{n}^{\circ} 2\right)$, « la même caractère » $\left(\mathrm{n}^{\circ} 5\right)$, « un conversation » $\left(\mathrm{n}^{\circ} 5\right)$, « un langue » $\left(\mathrm{n}^{\circ} 6\right)$, « une livre $»\left(\mathrm{n}^{\circ} 6\right)$, « un sœur » $\left(\mathrm{n}^{\circ} 6\right)$, « un mise à jour » $\left(n^{\circ} 7\right)$.

\section{Interférences syntaxiques}

Parmi les domaines d'interférence que nous avons mentionnés auparavant, seulement l'ordre et la constitution de la phrase, ainsi que le système des pronoms, ont été identifiés comme provoquant des interférences dans le discours de nos informatrices.

Des erreurs d'accord et de choix des prépositions ont également été commises par nos enquêtées, mais elles ne relèvent pas à notre avis d'interférences avec l'arabe, s'agissant plutôt de difficultés inhérentes au français.

En ce qui concerne les constituants de la phrase, nous avons repéré deux structures produites par nos informatrices qui semblent être des calques des structures arabes :

1. « je pense que là mon problème quoi, là où réside mon problème » $\left(\mathrm{n}^{\mathrm{o}} 1\right)$

2. « vous (i)maginez pas combien livres chez moi, combien-j’ai-de livres chez moi » $\left(\mathrm{n}^{\mathrm{o}} 6\right)$

Pour comprendre la première interférence, il faut tenir compte du fait que, en arabe, le verbe être n'est jamais exprimé en aspect inaccompli. Ainsi, une structure attributive comme le garçon est intelligent serait exprimée en arabe sans la copule : « le garçon intelligent » (əl-wald dki en arabe marocain) ${ }^{64}$. Dans notre exemple, l'enquêtée omet le verbe être aussi bien dans sa première formulation que dans la deuxième et transpose ainsi au français la structure de la phrase en arabe.

Dans le deuxième cas, il s'agit d'un calque de l'arabe maghrébin, où le mot Sandi (composé de la préposition Sand (auprès de) et le suffixe - $i$ de première personne du singulier) signifie à la fois j'ai et chez moi. L'enquêtée a donc visiblement omis l'un des deux éléments (j'ai), croyant qu'il était implicite dans l'autre, avant de s'en rendre compte et s'autocorriger.

Quant à l'ordre des mots, c'est la place de l'adverbe qui a attiré notre attention lors des entretiens. Étant donné que, en arabe, l'adverbe est normalement placé après le mot qu'il modifie, les exemples suivants pourraient relever d'une interférence à cet égard :

\footnotetext{
Cf. MARÇAIS, L'arabe maghrébin, 161.

Cf. MARÇAIS, L'arabe maghrébin, 163.

64 Cf. BAIDOUN, L'influence de la langue arabe, 36.
} 
1. « j’ai lu beaucoup » $\left(\mathrm{n}^{\circ} 6\right)$

2. « [...] qu'elles sont présents trop dans notre dialecte » $\left(\mathrm{n}^{\circ} 1\right)$

3. « on n'a pas appris assez » $\left(n^{\circ} 6\right)$

Toutefois, les règles concernant la place de l'adverbe en français s'avèrent confuses pour tous les étudiants, peu importe leur origine. Ces erreurs pourraient donc aussi être une conséquence des difficultés inhérentes au français, indépendamment des structures arabes.

Certains pronoms constituent une vraie source de difficultés pour les arabophones, comme le démontrent les études réalisées par Hamida ${ }^{65}$ et Baidoun ${ }^{66}$.

La confusion entre le pronom complément COD et COI, qui est brièvement exposée par Baidoun ${ }^{67}$, apparaît chez une de nos enquêtées : « je le demande [à son mari] qu'estce que ça veut dire cette mot? » $\left(\mathrm{n}^{\circ} 6\right)$. Cette formulation répond à une interférence du système des pronoms arabe, qui ne tient pas compte de leur fonction dans la phrase.

Mais ce sont les pronoms relatifs qui ont posé le plus de problèmes à nos enquêtées. Dans les propositions relatives arabes, la fonction n'est pas exprimée par le relatif comme en français, mais par le pronom personnel, qui est repris et qui renvoie à l'antécédent ${ }^{68}$. Ceci explique d'après Hamida « l'adjonction [en français] d'un pronom personnel redondant $»^{69}$.

Exemples :

1. « il y a des gens qu'ils connaissent pas la langue française » $\left(\mathrm{n}^{\circ} 6\right)$

2. «c'est elle qui... qu'elle a posé mon $\mathrm{CV} »\left(\mathrm{n}^{\mathrm{0}} 7\right)$

3. « quand tu dis un mot que c'est pas bon » $\left(\mathrm{n}^{\circ} 7\right)$

4. «[...] fière de mes origines, que je les oublie pas » $\left(\mathrm{n}^{0} 7\right)$

5. « [...] mots de français qu'elles sont présents $[\ldots] »\left(\mathrm{n}^{\circ} 1\right)$

Par ailleurs, les pronoms relatifs à fonction de complément prépositionnel semblent aussi supposer une difficulté pour nos enquêtées, à nouveau provoquée par l'absence de fonction des pronoms en arabe.

Exemples :

1. « j'ai des amis qui parlent en arabe, que je peux discuter quelques mots ou qu'on échange quelques mots » $\left(\mathrm{n}^{\circ} 5\right)$

2. «j'ai une amie française que je parle avec elle au téléphone $[\ldots] »\left(\mathrm{n}^{\circ} 6\right)$

Finalement, nous avons identifié deux possibles calques syntaxiques de la L1 de nos informatrices, tous les deux correspondant à un emploi fautif de l'article :

1. « je te remercie beaucoup, d'abord...euh... d'avoir la confiance en moi et donc... de me faire la confiance en fait $\gg\left(n^{\circ} 2\right)$

\footnotetext{
Cf. HAMIDA, « Erreurs interférentielles arabe-français ».

Cf. BAIDOUN, L'influence de la langue arabe.

Cf. BAIDOUN, L'influence de la langue arabe, 38.

68 Par ailleurs, en arabe maghrébin, le relatif ne varie pas en genre ni en nombre, même s'il a plusieurs formes possibles en fonction de ce que l'on désigne (une personne ou une chose) et de la région (cf. MARÇAIS, L'arabe maghrébin, 204-205).

69 HAMIDA, « Erreurs interférentielles arabe-français », 113.
} 
2. « à la force qu'ils parlent français $[\ldots] »\left(\mathrm{n}^{0} 7\right)$

Le premier exemple pourrait être un calque de l'arabe libyen, où l'on détermine (comme dans les autres dialectes du Maghreb) le mot confiance dans ce type de structures. Par exemple, la phrase en arabe libyen $\subseteq t^{\varsigma} u$ fi:ya t-ti:qa serait littéralement traduite en français par ils ont donné en moi la confiance. Le deuxième exemple, énoncé par une enquêtée marocaine, est possiblement un calque de l'expression marocaine $b$-əz-zazz, littéralement à la force.

\section{D'autres interférences}

Certains traits caractéristiques du discours de nos enquêtées, qui ne constituent pas des erreurs en soi, peuvent être identifiés comme des interférences de style. Ainsi, par exemple, une de nos enquêtées libyennes (la nº 2 ) abusait certainement de l'interjection d'accord, qu'elle employait même dans des situations où il est inhabituel de l'entendre, ce qui a attiré notre attention. En effet, en arabe libyen, l'interjection $b \bar{a} h i$ ou sa forme abrégée $b \bar{a} h$ sont très souvent employées dans les dialogues avec le même sens que d'accord, probablement avec une plus grande fréquence que l'interjection française.

Une autre interférence de style peut être remarquée dans la répétition des mots. Baidoun a déjà observé cette interférence, qui consiste d'après lui à « utiliser un mot plusieurs fois pour confirmer une idée $»^{70}$. L'informatrice $n^{\circ} 6 \mathrm{y}$ a recours à plusieurs reprises dans son discours.

Exemples :

1. «il fait des blagues des blagues tout le temps et moi j'ai arrêté de demander »

2. « [...] moi pendant le domaine esthétique, parce que j’ai bien parce que j’ai étudié bien bien bien que je trouve que je suis plus à l'aise à mon domaine »

3. « [...] je demande mon mari parce qu'il parle mieux que moi et toujours dans ma tête c'est [sifflement] bruit bruit, j'aimerais bien parler XXX comme on dit en anglais »

4. « [...] toujours je me sens que j'ai besoin j'ai besoin tout le temps j'ai besoin de XXX vocabulaire »

Enfin, la plupart de nos informatrices $\left(\mathrm{n}^{\circ} 1,2,4,5\right)$ alternent durant les entretiens le tutoiement et le vouvoiement, sans arriver à suivre une tendance fixe. Ceci est une difficulté inhérente à tous les locuteurs du français dont la L1 ne possède pas cette distinction dans la manière de s'adresser à son interlocuteur, comme c'est le cas des arabophones. Nous pourrions donc considérer cette difficulté et les incohérences qui en découlent comme une interférence sociolinguistique ${ }^{71}$.

70 BAIDOUN, L'influence de la langue arabe, 173. Le recours à la répétition des mots est aussi une caractéristique typique de l'oral en français, mais le fait qu'elle soit catégorisée comme interférence par un autre auteur nous incite à croire qu'il s'agit d'un recours plus fréquent en arabe. Par ailleurs, Ángeles Vicente, recueillant les propos exprimés par plusieurs dialectologues, note que la répétition pour donner de l'emphase est l'une des stratégies du discours féminin qui visent à affronter l'androcentrisme répandu dans la société marocaine (Cf. VICENTE, Ángeles, " Gender and language boundaries in the Arab World. Current issues and perspectives », dans Estudios de dialectología norteafricana y andalusí nº13 (2009), 7-30).

71 Cette catégorie d'interférence langagière est étudiée par Jarvis et Pavlenko dans Crosslinguistic influence. 


\section{L'interférence comme indice et déclencheur d'insécurité linguistique}

La non-maîtrise de la langue d'accueil est de toute évidence la cause principale de l'apparition du sentiment d'insécurité linguistique chez l'immigré. L'interférence étant, à son tour, souvent déclenchée par cette maîtrise insuffisante de la langue, il semble logique qu'il puisse exister une certaine relation de réciprocité entre l'interférence et le sentiment d'insécurité linguistique.

D'une part, certaines interférences constituent des indicateurs de l'existence de cette insécurité. En effet, parmi les interférences repérées dans notre corpus, il y en a deux qui renferment une volonté d'imiter des formes plus prestigieuses ou correctes et qui donnent lieu à des hypercorrections, comme nous l'avons déjà remarqué au cours de l'analyse du corpus. Ainsi, l'utilisation de /p/ au lieu de /b/ dans des mots comme poubelle témoigne d'une insécurité provoquée par la conscience de la difficulté à employer le phonème français /p/. Dans l'autre cas, c'est l'insécurité déclenchée par la différence de prestige entre l'arabe dialectal et le standard qui est à l'origine de l'interférence (revoir «Épenthèse provoquée par une hypercorrection », la dernière interférence phonologique décrite). Outre ces exemples, où la relation entre l'interférence et le sentiment d'insécurité est manifeste, le recours à des structures de la langue maternelle pourrait constituer en soi une stratégie involontaire pour surmonter l'insécurité linguistique : la locutrice introduit des éléments connus, qui représentent un terrain sûr et fiable, dans le discours dans une langue qui lui est étrange.

D'autre part, l'existence d'interférences dans le discours de nos informatrices semble être à son tour un déclencheur d'insécurité. Avoir conscience des difficultés entraînées par les différences entre les deux langues et de la possibilité de commettre une interférence met la locutrice dans un état d'alerte constant qui entraîne le doute. L'hésitation et la justification ultérieure de l'informatrice $\mathrm{n}^{\circ} 2$ font preuve de cette insécurité provoquée par la conscience de l'interférence : «ils m'ont dit à la lettre : on doit pas vous... vous lâcher, d'accord ? vous lâcher ou bien vous relâcher ? [rires] C'est ça le problème de loui, c'est ça la difficulté ici ». Cette relation entre l'interférence et l'insécurité peut également être observée dans l'alternance constante entre tutoiement et vouvoiement, qui est, comme nous l'avons commenté précédemment, présente dans le discours de la plupart de nos enquêtées. Conscientes que le vouvoiement n'existe pas dans leur langue maternelle et qu'elles risquent de tutoyer à tort, elles hésitent au moment d'utiliser le tutoiement et ne suivent pas une tendance constante ; elles tombent dans l'insécurité. C'est pour cette raison que, même si certaines d'entre elles ont affirmé préférer le tutoiement au début de l'entretien, le mélange a été présent tout au long de celui-ci.

\section{Conclusion}

L'influence de l'arabe dans le discours de nos enquêtées est remarquable, et ce dans tous les domaines de la langue. Les domaines qui révèlent une plus forte influence de la L1 de nos informatrices sont le phonologique et le morphologique, suivis par le domaine syntaxique, qui est loin d'être immunisé contre ce phénomène. Enfin, le lexique montre aussi, bien que dans une moindre mesure, des traits relevant d'une confusion entre les deux langues en contact.

Certaines des interférences repérées sont plus récurrentes que d'autres et apparaissent dans le discours d'un plus grand nombre d'enquêtées. Nous pouvons ainsi 
citer le remplacement des phonèmes $/ \mathrm{e} /, \mathrm{g} /$ et $/ \mathrm{y} / \mathrm{par} / \mathrm{i} /$ et de $/ \mathrm{b} / \mathrm{par} / \mathrm{p} /$; le choix erroné de l'article masculin ou féminin ; l'utilisation incorrecte des pronoms et le mélange entre le tutoiement et le vouvoiement comme étant les interférences les plus récurrentes (qui apparaissent dans le discours d'au moins la moitié de nos enquêtées).

L'informatrice qui a fait preuve d'une plus grande influence de l'arabe dans son discours est la $\mathrm{n}^{\circ} 6$, qui est d'ailleurs celle qui a un niveau de français plus bas. Elle a commis plus du double d'erreurs relevant d'interférences que les autres enquêtées et le domaine morphosyntaxique est celui dans lequel sa L1 fait irruption avec plus de force.

Une seule informatrice n'a pas commis d'erreur provoquée par une interférence (ni d'autre erreur que nous ayons perçue) : la $\mathrm{n}^{\circ} 3$. La ${ }^{\circ} 8$, bien qu'elle nous ait confirmé que le français était sa langue maternelle avec l'arabe, a fait preuve d'une petite influence de cette dernière dans le domaine phonologique. Il est donc intéressant de comparer la situation de ces deux enquêtées, la $\mathrm{n}^{\circ} 8$ ayant appris le français comme L1 et présentant de petites influences de l'arabe, alors que la $n^{\circ} 3$ n'est pas née dans un contexte bilingue, mais son français reste imperméable à l'influence de L1. Une possible explication à cette différence serait le fait que, pour la $n^{\circ} 8$, les deux langues étaient parlées dans un contexte arabophone, plus propice au mélange, tandis que les contextes francophone et arabophone étaient bien distincts pour la $\mathrm{n}^{\circ} 3$, pour qui le choix de la langue dépendait notamment du pays dans lequel elle se trouvait. Enfin, le reste d'enquêtées présente un degré similaire d'influence de la L1.

Cette étude fait donc preuve de la situation d'intime contact où se trouvent la langue arabe et la langue française, une situation dont ont déjà fait part d'autres études portant sur les interférences d'une langue sur l'autre et sur d'autres phénomènes dérivés du contact des langues comme le code-switching.

Finalement, nous avons constaté que le phénomène de l'interférence interagit de différentes manières avec le sentiment d'insécurité linguistique. D'une part, il agit en tant qu'indicateur et révélateur de l'existence de ce sentiment chez la locutrice ; d'autre part, il s'instaure en élément activateur, déclenchant l'hésitation chez la locutrice et, ainsi, son insécurité.

\section{Conventions de transcription}

[exemple] : commentaire externe à la transcription

$[\ldots]$ : coupure dans la transcription de l'enregistrement

XXX : séquence inaudible

ek-sem-ple : prononciation saccadée

exem(ple) : partie ou mot non prononcé mais sous-entendu

ex- : mot interrompu 